\section{Impfung verringert Risiko für grippeassoziierte Pneumonie}

\author{
Im Herbst und Winter stellt sich für viele Menschen die Frage \\ nach dem Für und Wider einer Grippeimpfung. Gerade bei \\ älteren Personen, chronisch Kranken und Schwangeren können \\ bei einer Erkrankung lebensbedrohliche Komplikationen \\ auftreten. Die Häufigkeit der grippeassoziierten Pneumonie \\ wurde nun bei Personen mit und ohne Impfschutz verglichen. \\ JAMA 2015; 314: 1488-1497
}

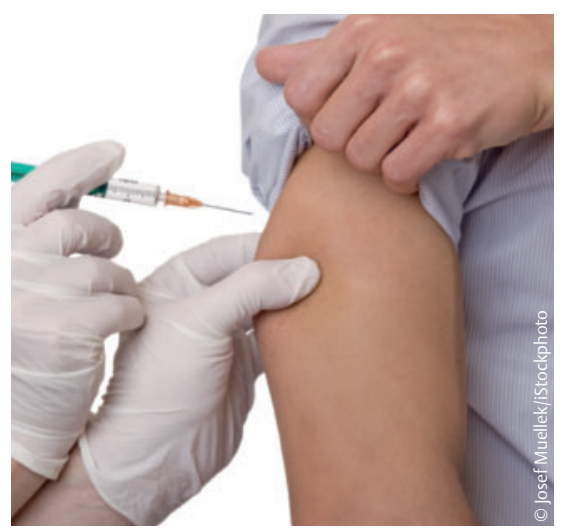

Die Grippeschutzimpfung wird allgemein als sinnvoll akzeptiert. Über ihre prinzipielle Effektivität gibt es in der Literatur kaum Zweifel. Erstaunlicherweise trifft dies nicht für die gefürchtete Komplikation der Influenzapneumonie zu. Unklar ist bisher, in welchem Umfang die Impfung auch das Risiko einer solchen, ambulant erworbenen Pneumonie reduziert.

C. G. Grijalva et al. werteten unter diesem Aspekt Daten einer prospektiven epidemiologischen Studie aus den USA (Etiology of Pneumonia in the Community, EPIC) in einem Fall-Kontroll-Setting neu aus. Es standen die Daten von 2767 Patienten mit ambulant erworbener Pneumonie zur Verfügung. Hiervon waren 794 (29\%) gegen Grippe geimpft. Die Forscher prüften dann, wie viele Geimpfte im Gegensatz zu Ungeimpften an einer Influenzapneumonie erkrankten. Alle Pneumoniefälle wurden stationär versorgt. Um die Influenzagenese der Pneumonie zu sichern, erfolgte bei Aufnahme ein Virusschnelltest. Säuglinge und Patienten mit unsicherem Impfstatus wurden nicht berücksichtigt. Der Beobachtungszeitraum erstreckte sich zwischen Januar 2010 und Juni 2012.
Als statistische Kontrollgruppe dienten die 2605 Patienten mit Pneumonie ohne Influenzagenese aus dem gleichen Studienkollektiv. Durch den Vergleich mit dieser Gruppe konnten die Autoren prüfen, welchen Einfluss ein positiver Impfstatus auf das Risiko hatte, eine Influenzapneumonie zu entwickeln. Damit konnte auch geklärt werden, in welchem Umfang die Impfung vor dieser Komplikation schützt, falls Patienten, trotz Impfung, an einer Influenza erkranken.

Bei 6\% der Geimpften wurde eine Influenzapneumonie nachgewiesen. Es dominierten die Typen H1N1 (38\%) und H3N2 (27\%). Die statistische Auswertung zeigte, dass eine Impfung das Risiko für die Influenzapneumonie mehr als halbierte (Odds Ratio 0,43). Der präventive Effekt war dabei stark vom Virustyp abhängig. Den höchsten Präventionserfolg hatten Patienten mit Influenzatyp B (Odds Ratio 0,28). Bei H1N1 lag dieser Risikowert bei 0,40 . Beim Virustyp H3N2 betrug die Risikoverminderung nur noch 0,55 .

\section{Fazit \\ Die Grippeschutzimpfung muss auch un- ter dem Aspekt des Schutzes vor Kompli- kationen propagiert werden. Kommt es trotz Impfung zu einer Erkrankung, dann wird das Risiko an einer Influenzapneu- monie zu erkranken drastisch vermin- dert. Patienten mit einer Erkrankung vom Influenza Typ B profitieren beson- ders vom Pneumonieschutz der Impfung.}

Dr. Horst Gross, Berlin

\section{Warum Rohmilch schützt}

Unverarbeitete Milch direkt vom Bauernhof hat einen höheren Omega-3-Fettsäuregehalt als pasteurisierte, homogenisierte und fettreduzierte Milch aus dem Laden. Das erklärt teilweise, warum RohmilchTrinker seltener Asthma entwickeln. Kinder, die Rohmilch trinken, entwickeln seltener Asthma als Kinder, die industriell verarbeitete Milch trinken. Dieser Effekt wurde bereits in mehreren Studien nachgewiesen und jetzt auch von LMU-Forschern im Rahmen der Pasture-Studie bestätigt. Wie die LMU-Forscher zugleich zeigen, liegt das u. a. am unterschiedlichen Gehalt von Omega-3-Fettsäuren in der Milch. Dennoch empfehlen sie nicht den Verzehr von Rohmilch, da diese krankmachende Mikroorganismen enthalten kann. Über ihre Ergebnisse berichten sie aktuell im Journal of Allergy and Clinical Immunology (DOI: http://dx.doi.org/10.1016/j. jaci.2015.10.042). Im Rahmen der Langzeitstudie PASTURE hielten rund 1000 Mütter Ernährung und Gesundheit ihres Kindes bis zum sechsten Lebensjahr regelmäßig fest. Das Risiko, mit 6 Jahren an Asthma zu erkranken, war bei jenen Kinder geringer, die ab der frühen Kindheit regelmäßig unverarbeitete Milch vom Bauernhof tranken. „Dieser Effekt lässt sich teilweise mit dem höheren Fettanteil und einem höheren Gehalt an Omega-3-Fettsäuren der Milch erklären“, sagt T. Brick aus der Arbeitsgruppe in München. Die Ergebnisse sind der Studie zufolge unabhängig von anderen möglichen Einflussfaktoren. Omega 3-Fettsäuren sind für den Menschen lebensnotwendig. Sie können vom Körper nicht selbst produziert und müssen mit der Nahrung aufgenommen werden. Ihnen werden verschiedene positive Wirkungen zugeschrieben. Die Forscher untersuchten verschiedene Arten von Milch: unverarbeitete und erhitzte Rohmilch sowie industriell verarbeitete Vollmilch bzw. fettreduzierte Milch. Je stärker die Milch verarbeitet war, desto geringer war der Gehalt an Omega-3- Fettsäuren. Der Gehalt an Omega-6-Fettsäuren, bei deren Abbau im Körper überwiegend entzündungsfördernde Derivate entstehen, veränderte sich jedoch kaum.

Nach einer Mitteilung der LudwigMaximilians-Universität München 